\title{
Observations of Solar Type III radio bursts by Cassini/RPWS experiment
}

\author{
Mohammed Y. Boudjada ${ }^{1}$, Ahmed Abou el-Fadl ${ }^{1}$, Patrick H. M. Galopeau ${ }^{2}$, Eimad Al-Haddad ${ }^{3}$, and \\ Helmut Lammer ${ }^{1}$ \\ ${ }^{1}$ Space Research Institute, Austrian Academy of Sciences, Graz, Austria \\ ${ }^{2}$ LATMOS-CNRS, Université Versailles Saint-Quentin-en-Yvelines, Guyancourt, France \\ ${ }^{3}$ University of Applied Sciences, Graz, Austria
}

Correspondence: Mohammed Y. Boudjada (mohammed.boudjada@oeaw.ac.at)

Received: 18 December 2019 - Revised: 28 July 2020 - Accepted: 27 August 2020 - Published: 10 December 2020

\begin{abstract}
In this study, we report on the occurrence of solar Type III radio bursts recorded by radio and plasma wave experiment (RPWS) experiment onboard Cassini spacecraft. This instrument is designed to investigate the Saturn's plasma environment and sub-auroral radio missions. RPWS/Cassini experiment allows to measure electric field over a frequency range from $1 \mathrm{~Hz}$ to $16 \mathrm{MHz}$ (Gurnett et al., 2004). The essential observed emission is associated to the Saturnian Kilometric Radiation (SKR) which is generated in the sub-auroral regions of the magnetosphere. The capability of this experiment leads to detect Solar Type III radio bursts recorded during the increase phase of the solar activity, i.e. January 2008 to December 2014. Hence we have found more than 300 Type III solar bursts when the distance of Cassini to the Sun was about $10 \mathrm{AU}$. Observational parameters like the time occurrence, the emission frequency and the relative intensity are considered in this analysis. Those features lead us to characterize the detection conditions and to estimate the occurrence variabilities of Type III bursts.
\end{abstract}

\section{Introduction}

The solar Type III radio bursts are generated by fast electron streams following opened magnetic field lines. Those electrons are considered to be ejected in the impulsive phase of a solar flare or solar-like event (Melrose, 1970). Langmuir waves in the solar corona and the interplanetary medium, which can be further converted to electromagnetic waves at the local plasma frequency, and/or its harmonic. It should be mentioned that the harmonic emission can better escape to the receiver in the interplanetary space than the fundamental one. Those radio bursts allow the remote sensing of the plasma environment in the vicinity of the Sun. A simple way to estimate the plasma frequency is to use the well-known relation between the electron density $d_{\mathrm{e}}\left(\mathrm{cm}^{-3}\right)$ and the local plasma frequency $f_{\mathrm{p}}(\mathrm{kHz})$, i.e. $d_{\mathrm{e}} \approx 9 f_{\mathrm{p}}^{1 / 2}$. Type III observed frequency $f_{\mathrm{o}}$ is supposed to be equal to the local plasma frequency or to one of its harmonics. Type III radio bursts can be observed at a large range of frequencies using ground based and space-borne observatories. They begin at a few hundred of megahertz and rapidly drift to low frequencies (i.e. about $30 \mathrm{kHz}$ ) which can be only observed from space. This drift from high to low frequencies when the time increases is directly related to the electron density decrease from the solar corona to the interplanetary medium. Heliospheric density models are derived from magnetohydrostatic equations (e.g., Mann et al., 1999) and also from empirical models based on Type III burst average speed along the Archimedean spiral (Leblanc et al., 1998), or on the estimation of the Type III frequency drift rates (e.g. Boudjada et al., 2006). Stereoscopic observations have allowed to localize the source regions and study the directivity of the emission beams related to Type III bursts. Dulk et al. (1996) showed that $68 \%$ solar bursts are observed by Wind and Ulysses spacecraft with a minimum of about $50 \%$ when Ulysses was close to the ecliptic and nearly directly behind the Sun. Using the observations from the same satellites, Bonnin et al. (2008) investigated the directivity of solar Type III bursts at the hectometric and kilometric wavelengths. Authors found that the full angular width of the diagram in longitude at the maximum $/ 10$ level is about $190^{\circ}$ in the frequency range 
$940-740 \mathrm{kHz}$, and increases to nearly $360^{\circ}$ at $104-80 \mathrm{kHz}$. Boudjada et al. (2014) assumed the emission diagram as a hollow cone with a variable opening angle depending on the radiation frequency. Authors showed that the opening angle should be of about $90^{\circ}$ at the decametric wavelengths.

In the Sect. 2 of this paper we focus to observational aspects of solar Type III burst at Saturn's orbit particularly the spectral pattern, the occurrence, the intensity variation and the frequency bandwidth. In Sect. 3, we discuss our main results and combined them to previous investigations. A summary and future perspectives are given in Sect. 4.

\section{Observational features of Solar Type III radio burst}

In this work we investigate solar Type III bursts recorded by the radio and plasma wave experiment (RPWS) onboard Cassini spacecraft. This instrument was designed to study the Saturn's plasma environment and sub-auroral radio missions. RPWS/Cassini experiment allowed to measure electric field over a frequency range from $1 \mathrm{~Hz}$ to $16 \mathrm{MHz}$ (Gurnett et al., 2004). We consider in this analysis the observations of the high frequency receiver (HFR) which is part of the Cassini/RPWS experiment. The temporal resolution was 32 seconds and the spectral one was $\Delta f / f=5 \%$ (Kurth et al., 2005). The usually observed emission is associated to the Saturnian Kilometric Radiation (SKR) which is generated in the sub-auroral regions of the magnetosphere. We consider solar bursts recorded by RPWS experiment in the time interval from the beginning of the year 2008 to the end of the year 2014. From the Type III spectral profile, we derive the observational parameters, i.e. the time and the corresponding frequency (in $\mathrm{kHz}$ ) and intensity level $(\mathrm{dB})$. Those parameters are combined to the Cassini positions (distance to the Sun in AU, latitude, longitude) expressed in the heliocentric system. Figure 1 displays dynamic spectrum recorded by RPWS experiment on 11 October 2013. The horizontal and vertical axes indicate, respectively, the observation time (in hours) and the frequency logarithmic scale (in kHz). Cassini spacecraft was at a distance of about $9.86 \mathrm{AU}$ with heliographic coordinate of $223^{\circ}$ longitude and $2.38^{\circ}$ latitude. The magnetospheric emission mainly appears at 10:45 UT in the frequency range between 8 and $100 \mathrm{kHz}$. The solar burst starts at 08:25 UT and ends at 10:00 UT and covers a larger bandwidth from about $16 \mathrm{MHz}$ and down to about $50 \mathrm{kHz}$. In Fig. 1, one can distinguish the solar bursts from SKR emissions. However the lower frequency of Type III is mainly overlapped by the SKR emissions. This means that we use the upper part of the RPWS frequency bandwidth, i.e. $1-16 \mathrm{MHz}$, to distinguish the solar burst. The relative intensity is principally indicated by red, green and blue colors corresponding to $25 \mathrm{~dB}$ (strong), $13 \mathrm{~dB}$ (medium) and $3 \mathrm{~dB}$ (low) levels. The background level is about $5 \mathrm{~dB}$. Hence the core part of the solar burst is saturated $(25 \mathrm{~dB})$ between $80 \mathrm{kHz}$ and $1 \mathrm{MHz}$, and then decreases to an intensity level

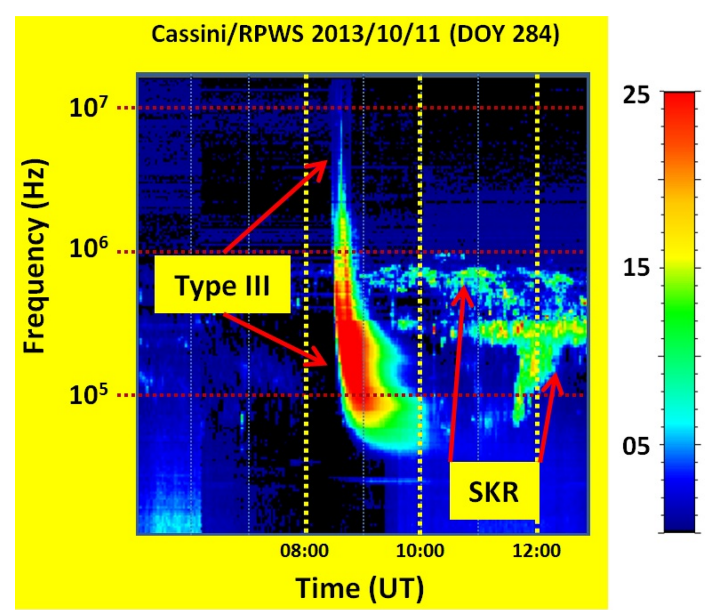

Figure 1. Dynamic spectrum of Solar Type III radio burst recorded by RPWS experiment on 11 October 2013. Solar burst is followed by Saturnian Kilometric Radiation (SKR). The intense and weak emissions correspond, respectively, to red and blue colors.

of about $13 \mathrm{~dB}$. In the range $2-3 \mathrm{MHz}$, the Type III intensity is very close to the background level. Generally the weakest intensity is recorded in the frequency range between 5 and $16 \mathrm{MHz}$. The frequency drift rate is found to be dependent on the frequency bandwidth, i.e. $-260,-0.2$ and $-0.003 \mathrm{kHz} \mathrm{s}^{-1}$ in the bandwidths $5-0.4,0.4-0.2$, and $0.2-$ $0.08 \mathrm{MHz}$.

\subsection{Type III occurrence}

We have considered the daily dynamic spectra recorded by RPWS experiment from 1 January 2008 to 31 December 2014. This period covers more than $2550 \mathrm{~d}$. We have found in this time interval a total of 325 Type III bursts occurring in the frequency range between $50 \mathrm{kHz}$ and $16 \mathrm{MHz}$. The occurrence probability of Type III burst is shown in Fig. 2, each bin corresponding to one observation day (i.e. $24 \mathrm{~h}$ ). We note an absence of solar burst from the beginning of the year 2008 to the middle of the year 2010. The first Type III was recorded on 25 July 2010, several months after the solar activity minimum in August 2008. Later on the solar bursts were regularly observed as shown in Fig. 2. However we remark a clear modulation of the occurrence with two minima around December-2010/January-2011 and May/June 2013 followed, respectively, with two maxima at about December2011/January-2012 and January 2014. The modulation period seems to be nearly equal to two years.

\subsection{Intensity level versus Type III Observed frequency}

We consider in Fig. 3 the variation of the intensity level versus the Type III burst frequencies. One can see a clear decrease of the intensity from about 20 to $3 \mathrm{~dB}$ when the frequency increases from about $90 \mathrm{khz}$ to $10 \mathrm{MHz}$. This is in 


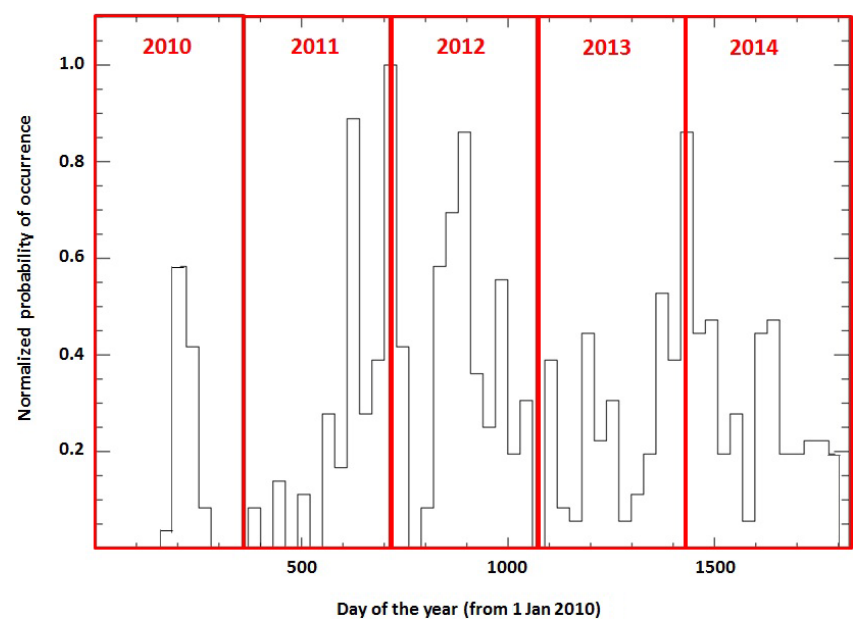

Figure 2. Occurrence of Solar Type III radio bursts versus day of the year (DOY) starting from 1 January 2010.

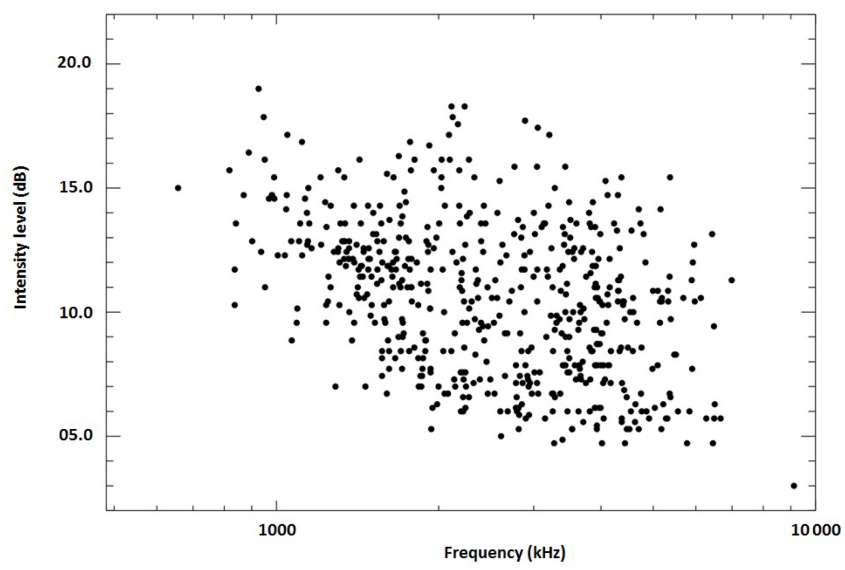

Figure 3. Variation of the intensity level versus the Type III observed frequency.

concordance with Type III burst displays in Fig. 1. Hence the higher frequencies of the burst between $2 \mathrm{MHz}$ and $16 \mathrm{MHz}$ are weaker than lower frequencies. Also, one note a large spread of values depending on the frequency. This effect may be estimated by taking into consideration the interval between the maximum and the minimum of the intensity level for a given frequency. We find that, on average, this interval is 8,13 and $10 \mathrm{~dB}$ when the Type III frequency is, respectively, 1,2 and $5 \mathrm{MHz}$. Above $2 \mathrm{MHz}$, the scattering points suggest a real difficulty to estimate the intensity level of the Type III solar bursts.

\subsection{Frequency occurrence probability}

The recorded Solar Type III bursts are principally found to occur at frequency below $3 \mathrm{MHz}$, as shown in Fig. 4. The kilometric and hectometric solar bursts are dominant and correspond to more than $70 \%$ of the total recorded Type III

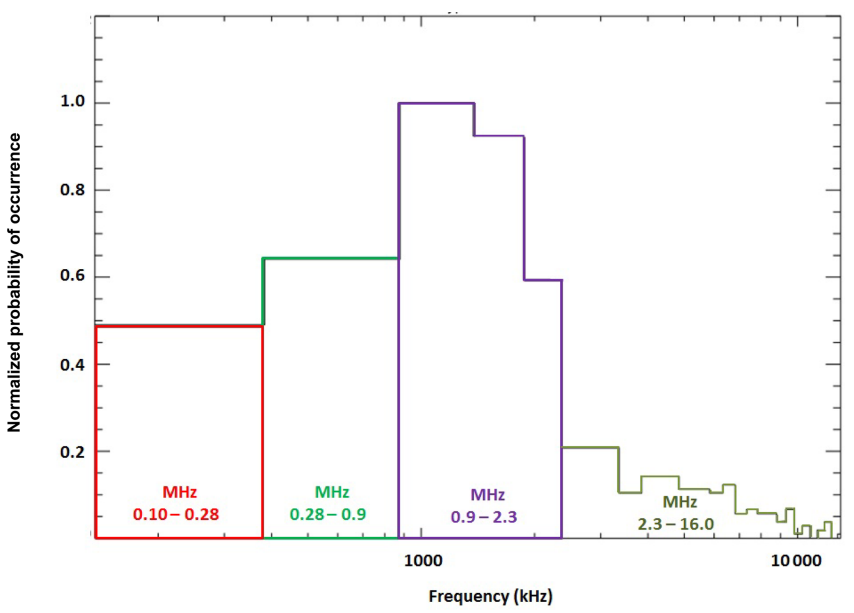

Figure 4. Occurrence of Solar Type III frequency bandwidth.

bursts. The frequency occurrence displays in Fig. 4 indicate the statistical distribution of the observed frequency derived from the Type III spectral profiles. The used bin is equal to $500 \mathrm{kHz}$ (i.e. $0.5 \mathrm{MHz}$ ), the frequency is given in logarithmic scale. The frequency occurrence increases, reach a maximum and then decreases, respectively, in the intervals $100 \mathrm{kHz}-1 \mathrm{MHz}, 1-2.5 \mathrm{MHz}$ and $2.5-16 \mathrm{MHz}$. The noticeable decrease of the occurrence at high frequency is clearly related to the low intensity level of Solar Type III bursts as one can deduce when combining Figs. 3 and 4. The maximum (i.e. $1-2.5 \mathrm{MHz}$ ) may be due to absence of Saturnian Kilometric Radiation above $1 \mathrm{MHz}$. This means that part of Solar bursts can't be detected when they are overlapped to SKR emissions.

\section{Discussion}

RPWS/Cassini experiment capabilities have allowed the detection of more than 300 Solar Type III radio bursts. Those are covering a large frequency range between $100 \mathrm{kHz}$ and $16 \mathrm{MHz}$. Hereafter we emphasize on the main results, the Type III burst occurrence for the investigated period (i.e. January 2008 to December 2014) and corresponding source locations in the Solar corona and the interplanetary medium.

\subsection{Detection of Type III bursts at $10 \mathrm{AU}$}

In this sub-section, we emphasize on the variability of the Type III occurrence. This occurrence variability is depending on the detection conditions associated to the reception system (i.e. antenna and receivers of Cassini/RWPS experiment), and the position and the distance of the observer (i.e. Cassini spacecraft) with regard to the Type III source regions. Hence detection conditions of Solar Type III bursts are found to exhibit minima and maxima of occurrence (Fig. 2). Several parameters may be considered to explain such vari- 
able occurrences. First parameter is the solar activity which reached its minimum in August 2008 until a maximum in the end of 2014. During two years (i.e. 2008 and 2009) no Type III bursts were recorded by RPWS experiment, and the first bursts appeared in the middle of 2010. Second parameter is the distance of about $10 \mathrm{AU}$ which separates the Sun from Cassini spacecraft. It is evident that the Type III radio flux is decreasing with the distance if we suppose an isotropic propagation. Third parameter is related to the previous one where the propagation is depending on the source location and the way electron beams are following opened solar magnetic field where the trajectory is a Parker spiral.

We compare our results with the one by Zhang et al. (2018) who investigated Type III bursts recorded by the Nançay Decameter Array (Aubier et al., 2000) in the period from 2012 to 2017. Those observations concern the frequency range from 10 to $80 \mathrm{MHz}$. Despite a different frequency bandwidth coverage, it is interesting to find similar occurrence variation of Type III bursts. In the first panel of Fig. 5 of Zhang et al. (2018) one see clearly minima and maxima particularly from 2012 to 2014 during the growth of the solar activity. Our investigations show similar behavior (i.e. Fig. 2) but alterations may be due to frequency bandwidths and observers (i.e. ground-based and space observations.

\subsection{Solar corona and interplanetary medium}

We have considered in this study more than 300 Type III bursts. We have shown that the dominant emission occurs at frequency lower than $2.3 \mathrm{MHz}$ (Fig. 4) which suggests Type III source localized in the interplanetary medium. Above $2.3 \mathrm{MHz}$, one note the lack of intense radio emission in the high frequency part of the Type III bursts. Two effects should be considered for the interpretation of the frequency occurrence. The first is associated to the emission source linked to Type III burst. Opening angle of the radiation cone is smaller at about $10 \mathrm{MHz}$ and increases to more than $180^{\circ}$ at lower frequency around $100 \mathrm{kHz}$. This means that the detection of Type III at hight frequency is strongly depending on the position of the observer (i.e. the spacecraft) with regard to the emission source, and to the distance between the observer and the source region. At low frequency $(\approx 500 \mathrm{kHz})$, stereoscopic observations leaded to show simultaneously observation of Type III bursts by three spacecraft (i.e. Wind, Ulysses and Cassini). Despite the different spacecraft locations, the spectral patterns were found to be similar by Boudjada et al. (2014). Also recent LOFAR investigations have shown the source regions in the solar corona at high frequency of Type III solar bursts. Mann et al. (2018) investigated dynamic imaging at decametric frequencies with a time resolution of $1 \mathrm{~s}$. Using radio imaging technique, the authors showed the spatial expansion of the Type III source region when the frequency increases from 60.55 down to $30.08 \mathrm{MHz}$. Peculiarly, Mann et al. (2018) found the source sizes increasing with propagation distance for decametric Type III bursts. At hectometric wavelengths, the source size covers a larger space which lead a better detections of Type III burst far from the Sun as investigated in this paper. The second effect occurs along the ray path propagation of the Solar burst. Refraction and scattering disturb the wave propagation because of the presence of plasma "anomalies". Poquerusse and McIntosh (1995) considered both effects in their study of the probability of occurrence of Type III during flares. Authors showed that in the case of the refraction, rays are generally bent towards low density regions. Thejappa et al. (2007) developed Monte Carlo simulation to study refraction and scattering effects associated, respectively, to spatial variation of the solar wind density and to random density fluctuations. Authors found that scattering effect is dominant at frequency of $120 \mathrm{kHz}$. At higher frequencies Thejappa and MacDowall (2008) showed combined effect where only refraction can occur or both effects (i.e. scattering and refraction). Authors concluded that such effects are at the origin of the unusual behavior of the radio emission of the quiet-Sun.

\section{Conclusion}

We have investigated the occurrence of Type III radio bursts recorded by Cassini/RPWS experiment during seven years, i.e. from 2008 to 2014. This occurrence is depending on the detection conditions associated to the reception system (i.e. Cassini/RWPS experiment), and also the position and the distance of the observer (i.e. Cassini spacecraft) with regard to the source regions. We have shown that the solar activity may play an important role in the detection of those solar bursts. However, the type III occurrence is found to be variable and exhibit maxima and minima of detections despite the enhancement phase of the solar activity. The intensity level is depending on the observed frequency where weak Type III emission is recorded at high frequency (i.e. above $10 \mathrm{MHz}$ ). The source regions of all those solar bursts are not automatically associated to the solar corona. Hence the dominant emission occurs at frequency lower than $2.3 \mathrm{MHz}$ which suggests Type III source localized in the interplanetary medium. In the near future, we will analyze the common Type III bursts simultaneously recorded by Cassini and Wind. The aim will be the estimation of the opening angle associated to the radiation cone of Type III radio bursts.

Data availability. The Cassini/RPWS data are available at The Planetary Plasma Interactions (PPI) Node of the Planetary Data System (PDS) archives on http://www-pw.physics.uiowa.edu/pds/css/ (PDS/PPI, 2020).

Author contributions. AAeF worked on the analysis of RPWS data, EAH provided a software for the data investigation, MYB prepared the manuscript with feedback from PHMG and HL. 
Competing interests. The authors declare that they have no conflict of interest.

Special issue statement. This article is part of the special issue "Kleinheubacher Berichte 2019". It is a result of the Kleinheubacher Berichte 2019, Miltenberg, Germany, 23-25 September 2019.

Acknowledgements. The authors are grateful to William S. Kurth (PI of the RPWS experiment) for making the data available for this work.

Review statement. This paper was edited by Gottfried Mann and reviewed by Pietro Zucca and two anonymous referees.

\section{References}

Aubier, A., Boudjada, M. Y., Moreau, P., Galopeau, P. H. M., Lecacheux, A., and Rucker, H. O.: Statistical studies of jovian decameter emissions observed during the same period by Nançay Decameter Array (France) and WAVES experiment aboardWind spacecraft, Astron. Astrophys., 354, 1101-1109, 2000.

Bonnin, X., Hoang, S., and Maksimovic, M.: The directivity of solar type III bursts at hectometer and kilometer wavelengths: WindUlysses observations, Astron. Astrophys., 489, 419-427, 2008.

Boudjada, M. Y., Lecacheux, A., Sawas, S., Stangl, A., and Voller, W. G.: Spectral study of solar Type III decametric bursts, in: Planetary Radio Emissions VI, edited by: Rucker, H. O., Kurth, W. S., and Mann, G., Austrian Academy of Sciences Press, Vienna, Austria, 401-408, 2006.

Boudjada, M. Y., Galopeau, P. H. M., Maksimovic, M., and Rucker, H. O.: Visibility of Type III burst source location as inferred from stereoscopic space observations, Adv. Radio Sci., 12, 167-170, https://doi.org/10.5194/ars-12-167-2014, 2014.

Dulk, G. A., Leblanc, Y., Bougeret, J.-L., and Hoang, S.: Type III bursts observed simultaneously by Wind and Ulysses, Geophys. Res. Lett., 23, 1203-1205, 1996.

Gurnett, D. A., Kurth, W. S., Kirchner, D. L., Hospodarsky, G. B., Averkamp, T. F., Zarka, P., Lecacheux, A., Manning, R., Roux, A., Canu, P., Cornilleau-Wehrlin, N., Galopeau, P., Meyer, A., Bostrom, R., Gustafsson, G., Wahlund, J.-E., Aahlen, L., Rucker, H. O., Ladreiter, H. P., Macher, W., Woolliscroft, L. J. C., Alleyne, H., Kaiser, M. L., Desch, M. D., Farrell, W. M., Harvey, C. C., Louarn, P., Kellogg, P. J., Goetz, K., and Pedersen, A.: The Cassini radio and plasma wave science investigation, Space Sci. Rev. J., 114, 395-463, 2004.
Kurth, W. S., Hospodarsky, G. B., Gurnett, D. A., Cecconi, B., Louarn, P., Lecacheux, A., Zarka, P., Rucker, H. O., Boudjada, M., and Kaiser, M. L.: High spectral and temporal resolution observations of Saturn kilometric radiation, Geophys. Res. Lett., 32, L20S07, https://doi.org/10.1029/2005GL022648, 2005.

Leblanc, Y., Dulk, G. A., and Bougeret, J.-L.: Tracing the electron density from the corona to $1 \mathrm{AU}$, rough the solar corona with LOFAR, Sol. Phys., 183, 165-180, 1998.

Mann, G., Jansen, F., MacDowall, R. J., Kaiser, M. L., and Stone, R. G.: A heliospheric density model and type III radio bursts, Astron. Astrophys. J., 348, 614-620, 1999.

Mann, G., Breitling, F., Vocks, C., Aurass, H., Steinmetz, M., Strassmeier, K. G., Bisi, M. M., Fallows, R. A., Gallagher, P., Kerdraon, A., Mackinnon, A., Magdalenic, J., Rucker, H., Anderson, J., Asgekar, A., Avruch, I. M., Bell, M. E., Bentum, M. J., Bernardi, G., Best, P., Birzan, L., Bonafede, A., Broderick, J. W., Brueggen, M., Butcher, H. R., Ciardi, B., Corstanje, A., de Gasperin, F., de Geus, E., Deller, A., Duscha, S., Eisloeffel, J., Engels, D., Falcke, H., Fender, R., Ferrari, C., Frieswijk, W., Garrett, M. A., Griessmeier, J., Gunst, A. W., van Haarlem, M., Hassall, T. E., Heald, G., Hessels, J. W. T., Hoeft, M., Hoerandel, J., Horneffer, A., Juette, E., Karastergiou, A., Klijn, W. F. A., Kondratiev, V. I., Kramer, M., Kuniyoshi, M., Kuper, G., Maat, P., Markoff, S., McFadden, R., McKay-Bukowski, D., McKean, J. P., Mulcahy, D. D., Munk, H., Nelles, A., Norden, M. J., Orru, E., Paas, H., Pandey-Pommier, M., Pandey, V. N., Pizzo, R., Polatidis, A. G., Rafferty, D., Reich, W., Roettgering, H., Scaife, A. M. M., Schwarz, D. J., Serylak, M., Sluman, J., Smirnov, O., Stappers, B. W., Tagger, M., Tang, Y., Tasse, C., ter Veen, S., Thoudam, S., Toribio, M. C., Vermeulen, R., van Weeren, R. J., Wise, M. W., Wucknitz, O., Yatawatta, S., Zarka, P., and Zensus, J. A.: Tracking of an electron beam through the solar corona with LOFAR, Astron. Astrophys. J., 611, A57, https://doi.org/10.1051/0004-6361/201629017, 2018.

Melrose, D. B.: On the theory of Type II and Type III solar radio bursts, Aust. J. Phys., 23, 871-884, 1970.

Poquerusse, M. and McIntosh, P. S.: Type III radio burst productivity of solar flares, Solar Phys. J., 159, 301-323, 1995.

Planetary Data System (PDS)/Planetary Plasma Interactions (PPI): Cassini/RPWS data, available at: http://www-pw.physics.uiowa. edu/pds/css/, last access: 16 August 2020.

Thejappa, G. and MacDowall, R .J.: Effects of scattering on radio emission from the quiet sun at low frequecies, Astrophy. J., 676, 1338-1345, 2008.

Thejappa, G., MacDowall, R. J., and Kaiser, M. L.: Monte Carlo simulation of directivity of interplanetary radio bursts, Astrophy. J., 671, 894-906, 2007.

Zhang, P. J., Wang, C. B., and Ye, L.: Type III radio burst automatic analysis system and statistic results for a half solar cycle with Nançay Decameter Array data, Astron. Astrophys. J., 618, A165, https://doi.org/10.1051/0004-6361/201833260, 2018. 